\title{
Vacuum-induced Stark shifts for quantum logic using a collective system in a high-quality dispersive cavity
}

\author{
A. Gábris ${ }^{1,3}$ and G. S. Agarwal ${ }^{2,3}$ \\ ${ }^{1}$ Research Institute of Solid State Physics and Optics, Hungarian Academy of Sciences, H-1525 Budapest P.O. Box 49, Hungary \\ ${ }^{2}$ Department of Physics, Oklahoma State University, Stillwater, Oklahoma 74078, USA \\ ${ }^{3}$ Physical Research Laboratory, Navrangpura, Ahmedabad 380009, India
}

(Received 17 November 2004; revised manuscript received 24 February 2005; published 13 May 2005)

\begin{abstract}
A collective system of atoms in a high-quality cavity can be described by a nonlinear interaction which arises due to the Lamb shift of the energy levels due to the cavity vacuum [Agarwal et al., Phys. Rev. A 56, 2249 (1997)]. We show how this collective interaction can be used to perform quantum logic. In particular we produce schemes to realize controlled-NOT gates not only for two-qubit but also for three-qubit systems. We also discuss realizations of Toffoli gates. Our effective Hamiltonian is also realized in other systems such as trapped ions or magnetic molecules.
\end{abstract}

DOI: 10.1103/PhysRevA.71.052316

PACS number(s): 03.67.Lx, 42.50.Pq, 32.80.Pj

\section{INTRODUCTION}

The possibility of doing quantum computation with neutral atoms is becoming more realistic with the advances in techniques relating to the trapping of few atoms which could even be addressed individually [1-3]. However, a number of experiments so far have been done with flying qubits [4-6] and a number of proposals exist on implementing quantum logic operations using cavity QED [7-11]. We note that the realization of a controlled-NOT gate between two qubits requires some form of interaction between the qubits. There are thus realizations which depend on the interaction between the center-of-mass degrees and the electronic degrees of freedom as in the case of ions [12-14], the interaction between the photonic qubit and the atom as in case of cavity QED [4]. Thus for doing logic operations with neutral atoms one would require an effective interaction between them. Note that we have to keep the distance between atoms such that selective addressing is possible for singe-qubit operations. On the other hand, if the atoms are far apart, then the electrostatic interaction between them is very weak. These problems can be overcome by using a high-quality dispersive cavity. It has been shown earlier that the interaction of trapped atoms with a single mode of the radiation field produces an effective interaction which can be utilized for doing quantum logic $[15,16]$. Though we shall work in the framework of this physical system, it is notable that the considered Hamiltonian is a special case of the Lipkin model [17], and similar Hamiltonians can be associated with the dynamics of ion traps [18] and $\mathrm{Fe}^{3+}$ ions of a large magnetic molecule [19].

In this paper we propose a technique to realize quantum computation using cavity QED based on a collective interaction between all qubits, and single-qubit rotations. We derive explicit results for systems of two and three atoms, and provide direct constructions of important quantum gates for both configurations. Our results imply that arbitrary quantum gates could be realized for the two and three qubit systems. In the latter case it is demonstrated by first deriving a universal two-qubit gate from the collective interaction of the three atoms. The two-qubit gate thus obtained consists solely of the collective three-atom interaction and single-qubit rotations. In particular, we do not use any techniques involving other auxiliary states to select pairs of atoms to interact, as it is common in the literature of cavity QED quantum computing [20-27]. Although this reduces the number of sources of decoherence, it renders the calculations for implementing universal gate sets more involved. It is more difficult since now we are constructing simpler gates such as the CNOT from the more complex three-qubit gates and single-qubit operations. We note that the major source of decoherence, the finite lifetime of cavity photons, is eliminated in this approximation because of the dispersive nature of the atomcavity interaction, and a finite loss rate $\kappa$ only reduces the strength of the collective interaction.

The outline of this paper is as follows. We shall introduce our system and qubits in Sec. II, present a brief summary of some key mathematical tools used during our calculations in Sec. III, then in Secs. IV and V we shall give specific constructions of controlled-NOT gates for $N=2$ and $N=3$ atoms, respectively. We discuss realizations of Toffoli gates in Sec. VI, and Sec. VII is dedicated to our conclusions.

\section{PHYSICAL SYSTEM}

We consider $N$ two-level atoms trapped in a cavity, with the atomic transition frequency $\omega_{0}$ detuned from the cavity resonance frequency $\omega$ by some value $\Delta$, and denote the dipole coupling between an atom and the cavity by $g$. The main source of decoherence in cavity systems is generally the relatively high loss rate of photons from the cavity. We introduce the parameter $\kappa$ to characterize this decay rate. Another significant source of decoherence is the spontaneous decay of the excited state to the ground state. Considering the number of many ways to work around this problem (e.g., deriving effective two-level atoms from a $\Lambda$-type system), we do not discuss this topic in the present paper, and the main result remains the demonstration of quantum computing in a dispersive cavity. We note, however, that for large enough detuning $\Delta$, the modification of the decay rate due to 
the Purcell effect becomes negligible; therefore spontaneous decay is not enhanced by the cavity. As the last assumption, to facilitate individual addressing of atoms we require that the atoms are well separated, i.e., their spatial wave functions are nonoverlapping.

It was shown in Ref. [15] that if the cavity is in a thermal state with mean photon number $\bar{n}$, tracing out for this cavity mode in the limit $g \sqrt{N} \ll|i \Delta+\kappa|$ results in a time evolution of the atoms that can well be approximated by a unitary process. To write the effective Hamiltonian corresponding to this evolution in a convenient form we introduce the following notations: Let the computational basis states $|0\rangle_{k}$ and $|1\rangle_{k}$ be defined as the ground $(|g\rangle)$ and excited $(|e\rangle)$ states of the $k$ th atom, respectively. Therefore we assign a qubit to each of the $N$ atoms trapped within the cavity. Then we take the collective spin- $N / 2$ operators

$$
S_{i}=\frac{1}{2} \sum_{k=1}^{N} \sigma_{i}^{(k)},
$$

with the $\sigma_{i}^{(k)}$ Pauli- $i$ operators $(i=x, y, z$ or,+-$)$ defined on the computational basis as usual. Now we may write the effective Hamiltonian for $N$ atoms with the collective spin operators $S_{i}$ and the total angular momentum square operator $S^{2}$ as

$$
\begin{aligned}
H_{N} & =\hbar \eta\left(S_{+} S_{-}+2 \bar{n} S_{z}\right) \\
& =\hbar \eta\left[S^{2}-S_{z}^{2}+(2 \bar{n}+1) S_{z}\right],
\end{aligned}
$$

where the coupling factor is $\eta=g^{2} \Delta /\left(\kappa^{2}+\Delta^{2}\right)$. We remark that since this system contains only the atoms, and the time evolution is Hamiltonian, the cavity losses are expected to play no role in decoherence. The decay through the factor $g^{2} \kappa /\left(\kappa^{2}+\Delta^{2}\right)$ would be rather small as we work in the limit $\Delta \gg \kappa$. Indeed the cavity lifetime typically affects the system only through the magnitude of the coupling factor $\eta$.

The main theme of this paper shall be to prove the universality of this interaction Hamiltonian. To this end we show that this way it is possible to generate all controlledNOT gates using only this interaction and single-qubit operations. To simplify our calculations we assume that the singlequbit operations can be carried out on a more shorter time scale than the period of the collective interaction. Therefore we regard single-qubit operations as instantaneous compared to the multiqubit operation generated by the collective Hamiltonian. The single-qubit rotations also serve as tools to control the interaction times required for a desired multiqubit gate based on similar principles as in standard NMR quantum computing [28-30].

Further simplification is applicable to the Hamiltonians (2) also. We shall drop terms linear in $S_{z}$ as these terms do not have any effect on the universality of $H$ because these correspond to single-qubit operations. More precisely, let

$$
R_{x, y, z}(\vartheta)=\exp \left(-i \vartheta \sigma_{x, y, z} / 2\right)
$$

denote standard $\mathrm{SU}(2)$ rotations. Since $S_{z}$ commutes with the rest of the Hamiltonian, it follows that the linear terms in Hamiltonians (2a) and (2b) may be effectively cancelled from the time evolution by applying $R_{z}(-2 \eta \bar{n} t)$ and
$R_{z}[-\eta(2 \bar{n}+1) t]$, respectively, to every qubit. It is important that the angle of rotation depends on the actual mean photon number (i.e., temperature) of the cavity, hence it must be known accurately. It also depends on the time $t$ for which $H_{N}$ is to be applied; however, as we shall see later, in the course of quantum logic gate operations this $t$ is known a priori. Also, because of these commutation properties this rotation can be carried out any time within the time window prescribed by $t$. Hence in this paper when we say that we work with Hamiltonians (2) without the linear terms, we assume that the linear terms are always compensated by appropriate single-qubit rotations.

\section{ENGINEERING TWO-QUBIT GATES}

For construction of desired two-qubit gates we have used a technique introduced in Ref. [31]. For conciseness we briefly summarize this technique.

We consider two two-qubit gates $M$ and $L$, with unit determinants. We term them equivalent if they can be transformed into each other using only single-qubit operations $O=O_{1} \otimes O_{2}$ and $O^{\prime}=O_{1}^{\prime} \otimes O_{2}^{\prime}$ as

$$
L=O^{\prime} M O \text {. }
$$

Here we used the tensorial product notation $\otimes$ to distinguish operators acting on different subsystems. A very important result of Ref. [31] is that this equivalence is perfectly characterized by two numbers: Let $M_{B}=Q^{\dagger} M Q\left(L_{B}=Q^{\dagger} L Q\right)$ with

$$
Q=\frac{1}{\sqrt{2}}\left(\begin{array}{cccc}
1 & 0 & 0 & i \\
0 & i & 1 & 0 \\
0 & i & -1 & 0 \\
1 & 0 & 0 & -i
\end{array}\right),
$$

a unitary rotation to a specific entangled basis related to the standard Bell states. Then defining $m=M_{B}^{T} M_{B}\left(l=L_{B}^{T} L_{B}\right)$ with the superscript $T$ denoting real transpose, the pairs $\left(\operatorname{Tr}^{2} m, \operatorname{Tr} m^{2}\right)$ and $\left(\operatorname{Tr}^{2} l, \operatorname{Tr} l^{2}\right)$ coincide if and only if $L$ and $M$ are equivalent according to Eq. (4).

A useful application of this definition is to use the matrices $m$ and $l$ to find the single-qubit operations $O$ and $O^{\prime}$ connecting two equivalent $M$ and $L$. The recipe is as follows: diagonalize $m(l)$, i.e., find $O_{M}\left(O_{L}\right)$ such that $m=O_{M}^{T} d_{M} O_{M}$ $\left(l=O_{L}^{T} d_{L} O_{L}\right)$ with $d_{M}\left(d_{L}\right)$ a diagonal matrix. Then the solution can be written as

$$
\begin{gathered}
O=O_{M} O_{L}^{T}, \\
O^{\prime}=O_{L}^{T} O^{T} M_{B}^{\dagger} .
\end{gathered}
$$

Generalization to nonunit determinant matrices gives

$$
\left[\operatorname{Tr}^{2} m / 16 \operatorname{det} M,\left(\operatorname{Tr}^{2} m-\operatorname{Tr} m^{2}\right) / 4 \operatorname{det} M\right]
$$

as invariants of $M$ (the constant factors are introduced for later convenience).

These results summarized by Eqs. (6) can be used to construct $L$ using $M$ only if $L$ and $M$ are equivalent. This is the similar problem as constructing controlled-NOT gates from controlled- $Z$ gates. There is, however, a more interesting 


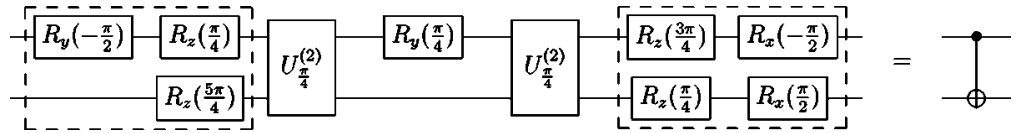

problem of constructing an $L$ using $A$ 's of a different equivalence class (e.g., SWAP gate using CNOT's). The invariants (7) can be used to tackle this problem also. Using invariants, we can split the problem into two, first searching for a matrix $M$ equivalent to our target $L$ in the form of $M=A O_{f} A$, and then using Eq. (6) to obtain $L$. This way we separate the nonlinear and the linear part of the problem, therefore gaining significant simplification over the conventional way of searching for a direct solution to $L$.

Unfortunately the invariants do not tell us much about the universality of a two-qubit gate $A$ and it is not known how many applications of $A$ are needed to reach a given $L$. For example a controlled-NOT gate (or any equivalent) may never be constructed from SWAP gates and single-qubit operations. Although throughout our calculations we could solve such problems with two applications of $A$, there may be cases when $A$ has to be used more than twice, e.g., $M$ $=A O_{f} A O_{f}^{\prime} A$.

\section{CASE OF $N=2$ ATOMS}

As the simplest case we consider two atoms in the cavity. The collective spin operators now describe a spin-1 system, and a number of simplifications apply to this case. For example, the Dicke states [32,33] span the complete Hilbert space of the two atoms and $G=\left[S^{2}-\left(S_{z}^{2}-S_{z}\right)\right] / 2$ is a projector operator. Considering Eq. (2a) without the linear terms we have $H_{2}=2 \hbar \eta G$, and the time-evolution operator is

$$
U^{(2)}(t)=e^{-(i / \hbar) H_{2} t}=1-e^{-i \eta t} 2 i G \sin (\eta t) .
$$

In the computational basis this corresponds to the matrix

$$
U^{(2)}(t)=e^{(-i \varphi)}\left(\begin{array}{cccc}
e^{-i \varphi} & 0 & 0 & 0 \\
0 & \cos \varphi & -i \sin \varphi & 0 \\
0 & -i \sin \varphi & \cos \varphi & 0 \\
0 & 0 & 0 & e^{i \varphi}
\end{array}\right)
$$

with $\varphi=\eta t$. The invariants (7) of this matrix are

$$
\left[\cos ^{4} \varphi, 4 \cos ^{2} \varphi-1\right]
$$

while for a controlled-NOT gate we would require $[0,1]$. We see that this requirement is not met by any real $\varphi$. After some algebra, however, we obtain that with $U_{\pi / 4}^{(2)}=U^{(2)}\left[(\pi / 4) \eta^{-1}\right]$, the sequence

$$
\tilde{U}^{(2)}=U_{\pi / 4}^{(2)} O_{f} U_{\pi / 4}^{(2)},
$$

is equivalent to a controlled-NOT gate if $O_{f}=R_{y}(\pi / 4) \otimes 1$. In particular, using this controlled-NOT equivalent gate $\tilde{U}^{(2)}$ a CNOT with first bit as control and second as target bit can be produced as

$$
U_{\mathrm{CNOT}}=e^{i \pi / 4} O_{c}^{\prime} \widetilde{U}^{(2)} O_{c},
$$

the single-qubit operations of this formula being
FIG. 1. Quantum circuit diagram depicting the sequence to prepare controlled-NOT gate from the time-evolution operator in Eq. (9).

$$
\begin{gathered}
O_{c}^{\prime}=\left[R_{x}(-\pi / 2) R_{z}(3 \pi / 4)\right] \otimes\left[R_{x}(\pi / 2) R_{z}(\pi / 4)\right], \\
O_{c}=\left[R_{z}(\pi / 4) R_{y}(-\pi / 2)\right] \otimes R_{z}(5 \pi / 4) .
\end{gathered}
$$

The phase factor is in principle irrelevant and is written there for didactic reasons only. The construction is depicted as a quantum circuit diagram in Fig. 1. We note here that assuming two-qubit gates $U^{(2)}$ with equal $t$, this construction is optimal in terms of operation time for the complete controlled-NOT gate.

\section{CASE OF $N=3$ ATOMS}

In contrast to the $N=2$ atoms case when collective interaction is a pair interaction itself, now the collective nature of the interaction is more pronounced as it involves all three qubits simultaneously. Since the invariant method of Sec. III has not been generalized to three-qubit gates yet, we first derive a two-qubit gate using single qubit operations, i.e., a gate which can be written as

$$
U_{23}^{(3)}=1 \otimes U_{23} .
$$

Therefore we can again use the invariants in the subsequent calculations involving $U_{23}$. This approach seems more efficient than trying to directly construct a collective quantum gate such as the Toffoli or Fredkin gate for three atoms.

For the calculations in this section we consider $\mathrm{H}_{3}$ of Eq. (2b) without the linear terms. To begin we use this interaction to generate two-qubit quantum gates of the form (15). Following the scheme similar to the spin-echo technique, we search for operators fulfilling Eq. (15) in the form

$$
U_{23}^{(3)}(t)=X_{1} U^{(3)}(t) X_{1} U^{(3)}\left(t^{\prime}\right) .
$$

Here $U^{(3)}(t)=\exp \left[-(i / \hbar) t H_{3}\right]$ is the time evolution generated by the chosen Hamiltonian, and $X_{1}=R_{x}(\pi) \otimes 1 \otimes 1$, which is essentially a NOT gate. We pose the condition (15) on Eq. (16) to find the appropriate $t$ and $t^{\prime}$.

The time evolution operator $U^{(3)}(t)$ is diagonal in the Dicke-state basis. To develop further insight into the problem, we apply the theory of angular momentum addition, and separate our spin-3/2 system into a product of a spin- $1 / 2$ and a spin-1 subsystem. The elements in the transformation matrix to the product basis are given by the relevant Clebsch-Gordan coefficients.

We require that Eq. (16) act on the spin-1/2 subsystem as the identity. This condition translates to $t^{\prime}=t$ and $\sin (3 / 2 \eta t)=0$, giving three distinct solutions for $U_{23}$ for each $i=-1,0,1$ via $\eta t=2 / 3 \pi(3 k+i)(k \in \mathbb{Z})$. Out of these three, $i$ $=0$ corresponds to the identity operator and is therefore irrelevant. The solutions for $i=-1$ and $i=1$ are adjoint of one another, also they are equivalent in the sense of Eq. (4) as they both have invariants $[1 / 4,3 / 2]$. In the following we work out the controlled-NOT gate explicitly for $i=1$, because we may expect shorter gate operation times. 
Our two-qubit unitary is represented by

$$
U_{23}=\left(\begin{array}{cccc}
e^{-i \pi / 3} & 0 & 0 & 0 \\
0 & e^{i \pi / 3} & 0 & 0 \\
0 & 0 & e^{i \pi / 3} & 0 \\
0 & 0 & 0 & e^{-i \pi / 3}
\end{array}\right)
$$

in the computational basis. We note that Eq. (17) may be written as $\exp \left(-i \pi / 3 \sigma_{z} \otimes \sigma_{z}\right)$ resembling the Heisenberg spin-spin interaction that has found many applications in quantum information processing, most notably NMR quantum computing [28-30]. However, in this case the interaction time is fixed by the conditions on $t$ and $t^{\prime}$ of Eq. (16). Nevertheless, we show that it is possible to express controlled-NOT gates using this operator and single-qubit gates.

Having a well-defined two-qubit gate in hand we turn to the technique of Sec. III. After straightforward algebra we find that using the single-qubit operators acting on the subspace of qubits 2 and 3 ,

$$
\begin{gathered}
O_{f}=1 \otimes R_{y}\left(\varphi_{f}\right), \\
O_{c}=R_{x}(-\pi / 2) \otimes\left[R_{z}(\pi) R_{x}\left(\varphi_{c}\right)\right], \\
O_{c}^{\prime}=\left[R_{z}(-\pi / 2) R_{y}(-\pi)\right] \otimes\left[R_{z}\left(\varphi_{c}^{\prime}\right) R_{y}(-\pi / 2) R_{z}(\pi / 2)\right],
\end{gathered}
$$

with

$$
\begin{gathered}
\tan \left(\varphi_{f} / 2\right)=1 / \sqrt{2}, \\
\tan \left(\varphi_{c} / 2\right)=\sqrt{2 / 3}-1, \\
\tan \left(\varphi_{c}^{\prime} / 2\right)=(1-\sqrt{3}) / \sqrt{2},
\end{gathered}
$$

the controlled-NOT gate can be constructed as

$$
U_{\mathrm{CNOT}}=e^{-i \pi / 4} O_{c}^{\prime} U_{23} O_{f} U_{23} O_{c} .
$$

This controlled-NOT gate acts on qubits 2 and 3 as control and target bits, respectively. However, due to the symmetry of $H_{3}$, a controlled-NOT gate acting the other way around or connecting different qubits is achievable simply by exchanging the roles of qubits appropriately with respect to our single-qubit operations.

\section{TOFFOLI GATES}

Universality of controlled-NOT gates implies that having them in all configurations for three qubits allows the construction of any three-qubit quantum gate, i.e., any $\operatorname{SU}\left(2^{3}\right)$ operator. In this section we demonstrate that it is possible to construct collective gates for all three qubits using our collective interaction. As a practical example we consider an important building block for systematic construction of complex quantum circuits, the Toffoli gate [34]. We also discuss a simplified version of the Toffoli gate (Fig. 2) that differs from the Toffoli gate only in one conditional phase shift

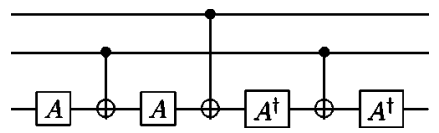

FIG. 2. Quantum circuit diagram for the simplified Toffoli gate requiring only three controlled-NOT gates $[34]\left[A=R_{y}(\pi / 4)\right]$.

whereas requiring only half the controlled-NOT gates. In certain quantum circuits some Toffoli gates may be replaced by the simplified versions without altering the effect of the overall circuit.

Since the decomposition of both these important gates into CNOT's is known, we can use our previous expressions of CNOT, and substitute them into the quantum circuits. Simple arithmetic counting the number of applications of $U^{(3)}\left(2 \pi / 3 \eta^{-1}\right)$ operations gives the gate times for the Toffoli and its simplified version. These operation times add up to $16 \pi / \eta$ and $8 \pi / \eta$ for the Toffoli and the simplified Toffoli gates, respectively. Following DiVincenzo's criteria [35], for efficient error-free quantum computation these gate times should be much shorter than the coherence time of the complete system.

\section{CONCLUSIONS}

In this paper we have shown the computational universality up to three qubits of a cavity assisted interaction between two-level atoms trapped in a dispersive cavity. We have proposed to use this collective interaction and single-qubit operations to implement two and three-qubit gates directly, rather than by selectively switching "on" and "off" the interaction between pairs of atoms. Therefore we do not require any additional levels, and this gives a greater flexibility for future generalization. In addition to the collective interaction we only need single-qubit operations to implement multiqubit gates. This requires that the atoms are separately addressable, and we also assume that single-atom operations can be performed on much shorter time scales than the collective interaction. The formalism used is not specific to two or three atom systems and therefore allows for further generalizations to more qubits.

In comparison with most previous proposals, since we do not excite the cavity during gate operations our scheme is robust against cavity decay. Also, dealing with thermal cavity states is made very straightforward within this theoretical framework. We believe therefore that this scheme may find useful applications in situations where good localization of atoms had been achieved inside a cavity.

\section{ACKNOWLEDGMENTS}

This work was partly supported by the National Research Fund of Hungary under Contract Nos. T 034484 and T 043079; the Marie Curie Programme of the European Commission; and by the Hungarian Ministry of Education under Contract No. CZ-5/03. A.G. would also like to thank the Director of P.R.L., Ahmedabad for his warm welcome and for all his support, and J. Janszky for useful discussions. 
[1] P. Hyafil, J. Mozley, A. Perrin, J. Tailleur, G. Nogues, M. Brune, J. M. Raimond, and S. Haroche, Phys. Rev. Lett. 93, 103001 (2004).

[2] W. Hänsel, P. Hommelhoff, T. W. Hänsch, and J. Reichel, Nature (London) 413, 498 (2001).

[3] J. McKeever, J. R. Buck, A. D. Boozer, A. Kuzmich, H.-C. Nagerl, D. M. Stamper-Kurn, and H. J. Kimble, Phys. Rev. Lett. 90, 133602 (2003).

[4] A. Rauschenbeutel, G. Nogues, S. Osnaghi, P. Bertet, M. Brune, J. M. Raimond, and S. Haroche, Phys. Rev. Lett. 83, 5166 (1999).

[5] Q. A. Turchette, C. J. Hood, W. Lange, H. Mabuchi, and H. J. Kimble, Phys. Rev. Lett. 75, 4710 (1995).

[6] P. Domokos, J. M. Raimond, M. Brune, and S. Haroche, Phys. Rev. A 52, 3554 (1995).

[7] T. Pellizzari, S. A. Gardiner, J. I. Cirac, and P. Zoller, Phys. Rev. Lett. 75, 3788 (1995).

[8] P. Milman, H. Ollivier, F. Yamaguchi, M. Brune, J. M. Raimond, and S. Haroche, J. Mod. Opt. 50, 901 (2002).

[9] A. Biswas and G. S. Agarwal, Phys. Rev. A 69, 062306 (2004).

[10] M. O. Scully and M. S. Zubairy, Phys. Rev. A 65, 052324 (2002).

[11] A. Biswas and G. S. Agarwal, Phys. Rev. A 70, 022323 (2004).

[12] M. D. Barrett et al., Nature (London) 429, 737 (2004).

[13] M. Riebe et al., Nature (London) 429, 734 (2004).

[14] B. DeMarco et al., Phys. Rev. Lett. 89, 267901 (2002).

[15] G. S. Agarwal, R. R. Puri, and R. P. Singh, Phys. Rev. A 56, 2249 (1997).

[16] S.-B. Zheng, Phys. Rev. A 68, 035801 (2003).

[17] H. J. Lipkin, N. Meshkov, and A. J. Glick, Nucl. Phys. 62, 188
(1965).

[18] R. G. Unanyan and M. Fleischhauer, Phys. Rev. Lett. 90, 133601 (2003).

[19] E. Keçecioğlu and A. Garg, Phys. Rev. B 63, 064422 (2001).

[20] S. J. van Enk, J. I. Cirac, and P. Zoller, Phys. Rev. Lett. 79, 5178 (1997).

[21] X. X. Yi, X. H. Su, and L. You, Phys. Rev. Lett. 90, 097902 (2003).

[22] E. Solano, M. Franca Santos, and P. Milman, Phys. Rev. A 64, 024304 (2001).

[23] M. Feng, Phys. Rev. A 66, 054303 (2002).

[24] E. Jané, M. B. Plenio, and D. Jonathan, Phys. Rev. A 65, 050302(R) (2002).

[25] J. Pachos and H. Walther, Phys. Rev. Lett. 89, 187903 (2002).

[26] C.-P. Yang, Shih-I Chu, and S. Han, Phys. Rev. A 70, 044303 (2004).

[27] L. You, X. X. Yi, and X. H. Su, Phys. Rev. A 67, 032308 (2003).

[28] I. L. Chuang, L. M. K. Vandersypen, X. Zhou, D. W. Leung, and S. Lloyd, Nature (London) 393, 143 (1998).

[29] D. G. Cory, M. D. Price, and T. F. Havel, Physica D 120, 82 (1998).

[30] T. S. Mahesh, N. Sinha, K. V. Ramanathan, and A. Kumar, Phys. Rev. A 65, 022312 (2002).

[31] Y. Makhlin, Quantum Inf. Process. 1, 243 (2002).

[32] R. H. Dicke, Phys. Rev. 93, 99 (1954).

[33] F. T. Arecchi, E. Courtens, R. Gilmore, and H. Thomas, Phys. Rev. A 6, 2211 (1972).

[34] A. Barenco, C. H. Bennett, R. Cleve, D. P. DiVincenzo, N. Margolus, P. Shor, T. Sleator, J. A. Smolin, and H. Weinfurter, Phys. Rev. A 52, 3457 (1995).

[35] D. P. DiVincenzo, Fortschr. Phys. 48, 771 (2000). 